\title{
Infective endocarditis - a critical review
}

\author{
Infective endocarditis, dentistry and antibiotic prophylaxis; time for a rethink? by R. A. Seymour, R. Lowry, \\ J. M. Whitworth, and M. V. Martin Br Dent J 2000; 189: 610-616
}

\section{Objective}

To provide a critical review of the current evidence that links dental treatment to infective endocarditis (IE) and appraise the risks of antibiotic chemoprophylaxis.

\section{Design \\ Retrospective analysis}

\section{Setting}

Mainly hospital based patients or subjects

\section{Outcome measures}

The interrelationship between infective endocarditis and dental treatment is complex and in many instances uncertain. The risk from antibiotic chemoprophylaxis appear greater than the risk of contracting IE.

\section{Results \\ There is increasing evidence that spontaneous bacteraemia are more likely to cause IE in at risk patients than specific episodes of dental treatment. Antibiotic chemoprophylaxis may not necessarily reduce dental-induced bacteraemia and the protective effect if any from antibiotic cover may arise from an inhibitory action upon bacterial colonisation on the compromised cardiac valves.}

\section{Conclusion}

There is increasing concern over the misuse of antibiotics in general and this has focused attention on chemoprophylaxis in dentistry to prevent IE. New evidence on dental-induced bacteraemia and the prevalence of IE in association with dental treatment raises further questions on the need to provide antibiotic cover in at risk patients. More prescriptive guidelines to define who is at risk from IE and what procedures require cover will help to reduce overprescribing of antibiotics and reduce the risks of their unwanted effects.

\section{Comment}

The precise value of antibiotic chemo1 propylaxis in the prevention of infective endocarditis has been questioned for over 16 years. $^{1,2}$ The question raised by Seymour et al. is important as there now appears to be a divergence of opinion between the American Heart Association (AHA) guidelines published in 1997, ${ }^{3}$ and the UK guidelines published in 1993 which have not been comprehensively updated since that time. 4

This difference in approach raises questions as to the appropriateness of the current guidelines of both the AHA and the UK working parties. This is especially worrying as there is little or no objective evidence that antibiotic prophylaxis is effective in preventing infective endocarditis in humans.

There is now considerable evidence of the differential susceptibility of groups of patients with predisposing cardiac lesions. There is further concern over the widespread use of antibiotics which may be ineffective and whose unwarranted use may contribute to the increasing pool of antibi- otic resistant organisms in the community. It is these considerations that have led the above authors to call for a rethink.

The authors cite the large amount of data available for the prevalence of bacteraemia following a variety of dento-gingival manipulative procedures with chewing and oral hygiene practices providing the greatest risk of cumulative bacteraemia. ${ }^{5}$

The quantifiable risks of adverse reactions to antibiotics is also alluded to. Whether the life threat to patients 'at risk' from infective endocarditis is greatest from the drugs used to prevent the endocarditis or from the risk of endocarditis following unprotected dental treatment is in need of a quantitative evaluation. A recent report from the USA would suggest that the risk of IE following dental treatment is virtually nil. ${ }^{6}$

Is it time for a rethink? Yes! but one that must be careful and comprehensive. The Endocarditis Working Party, an august body whose deliberations affect the lives of many thousands of patients and dentists in the UK and overseas, must be given time to consider these issues and reconcile the many differing views between individual research workers and advisory bodies

1 Guntheroth W G. How important are dental procedures as a cause of infective endocarditis. AmJ Cardiol 1984; 54: 797-801.

2 Clemens J D, Ransohoff D F. A quantitative assessment of pre-dental antibiotic prophylaxis for patients with mitral valve prolapse. J Chron Dis 1984; 37: 531-534.

3 Dajani A S, Taubert K A and Wilson W et al. Prevention of bacterial endocarditis. Recommendations of the American Heart Association. J Am Med Ass 1997; 277: 1794-1801.

4 Endocarditis Working Party of The British Society of Antimicrobial Chemotherapy. Recommendations for endocarditis prophylaxis. J Antimicrob Chemoth 1993; 31: 437-438.

5 Roberts G I. Dentists are innocent! 'Everyday' bacteraemia is the real culprit: A review and assessment of the evidence that dental surgical procedures are the principal cause of bacterial endocarditis in children. Ped Cardiol 1999; 35: 311-315.

6 Strom B L, Abrutyn E, Berlin J A et al. Dental and cardiac risk factors for infective endocarditis. Ann Int Med 1998; 729: 761-769.

\section{Graham J Roberts}

Joint Professor of Paediatric Dentistry, The

Eastman Dental Institute and The Institute of

Child Health 